\title{
CHARACTERISTIC NUMBERS OF RATIONAL CURVES WITH CUSP OR PRESCRIBED TRIPLE CONTACT
}

\author{
JOACHIM KOCK*
}

\begin{abstract}
This note pursues the techniques of [Graber-Kock-Pandharipande] to give concise solutions to the characteristic number problem of rational curves in $\mathrm{P}^{2}$ or $\mathrm{P}^{1} \times \mathrm{P}^{1}$ with a cusp or a prescribed triple contact. The classes of such loci are computed in terms of modified psi classes, diagonal classes, and certain codimension- 2 boundary classes. Via topological recursions the generating functions for the numbers can then be expressed in terms of the usual characteristic number potentials.
\end{abstract}

\section{Introduction}

With the advent of stable maps and quantum cohomology (Kontsevich-Manin [11]), there has been a tremendous progress in enumerative geometry. One subject of much research activity has been the characteristic number problem, notably for rational curves. Highlights of these developments include Pandharipande [13], who first determined the simple characteristic numbers of rational curves in projective space; Ernström-Kennedy [5] who computed the numbers for $\mathrm{P}^{2}$ using stable lifts - a technique that also allowed to determine characteristic numbers including a flag condition, as well as characteristic numbers of cuspidal plane curves; and Vakil [16] who used degeneration techniques to give concise recursions for the characteristic numbers also for elliptic curves.

With the notions of modified psi classes and the tangency quantum potential introduced in Graber-Kock-Pandharipande [7], conceptually simpler solutions were given to the characteristic number problem for rational curves in any projective homogeneous space, as well as for elliptic curves in $\mathrm{P}^{2}$ or $\mathrm{P}^{1} \times$ $P^{1}$. Tangency conditions allow simple expressions in terms of modified psi classes, and then the solutions follow from standard principles in GromovWitten theory, e.g. topological recursion.

Having settled the question of characteristic numbers of nodal rational curves, a natural next problem to consider is that of cuspidal curves, or to impose

\footnotetext{
* Supported by the National Science Research Council of Denmark.

Received March 5, 2001.
} 
higher order contacts, e.g. specified flex lines. In the 1870's, Schubert [15] computed the characteristic numbers of cuspidal plane cubics, and in the 1980's, a lot of work was devoted to the verification of his results, cf. Sacchiero [14], Kleiman-Speiser [8], Miret-Xambó [12], and Aluffi [1].

The techniques of stable lifts allowed L. Ernström and G. Kennedy [5] to determine the characteristic numbers of plane rational curves with cusp for any degree, and their joint paper with S. J. Colley [3] represents a big advance in the treatment of third order contacts.

The present note shows how the techniques of modified psi classes developed in Graber-Kock-Pandharipande [7] (henceforth cited as [GKP]), can also be used to solve the characteristic number problem for cuspidal rational curves in $\mathrm{P}^{2}$ or $\mathrm{P}^{1} \times \mathrm{P}^{1}$ (as well as that of a single triple contact to a given curve). To this end, a slight generalisation of the tangency quantum potential is needed, namely incorporating invariants corresponding to top products one factor of which is a square of a modified psi class or a certain codimension-2 boundary class. Via certain topological recursions, these new potentials are related to the usual tangency quantum potential. The locus of cuspidal curves and the locus of curves with a triple contact are now described in terms of these classes, and thus the corresponding generating functions can be expressed in terms of the slightly enriched potentials. In this way, the solutions to the corresponding counting problems come out rather easily, illustrating the versatility of the methods of [GKP].

The corresponding recursions have been implemented in maple; code or numerical data is available upon request.

The material of this note constituted Chapter 4 of my $\mathrm{PhD}$ thesis [9], and it is a pleasure here to thank the Departamento de Matemática da Universidade Federal de Pernambuco for four lovely years, and in particular my advisor Israel Vainsencher for his guidance and encouragement. I have also profited from conversations with Letterio Gatto and Lars Ernström.

\section{Preliminaries}

\subsection{The target space}

Throughout we work over the field of complex numbers. Let $X$ denote a projective homogeneous variety, and let $T_{0}, \ldots, T_{r}$ denote the elements of a homogeneous basis of the cohomology space $H^{*}(X, \mathrm{Q})$. In the applications $X$ will be $\mathrm{P}^{2}$ or $\mathrm{P}^{1} \times \mathrm{P}^{1}$. Let $g_{i j}$ denote the Poincaré metric constants $\int_{X} T_{i} \cup T_{j}$; we set also $g_{i j k}=\int_{X} T_{i} \cup T_{j} \cup T_{k}$. Let $\left(g^{i j}\right)$ be the inverse matrix to $\left(g_{i j}\right)$. It is used to raise indices as needed; in particular, with $g_{i j}^{k}=\sum_{e} g_{i j e} g^{e k}$, we can write $T_{i} \cup T_{j}=\sum_{k} g_{i j}^{k} T_{k}$. 


\subsection{The deformed metric}

(See Kock [10] for details.) Let $\mathbf{y}=\left(y_{0}, \ldots, y_{r}\right)$ be formal variables, and put

$$
\phi=\sum_{\mathbf{s}} \frac{\mathbf{y}^{\mathbf{s}}}{\mathbf{s} !} \int_{X} \mathbf{T}^{\mathbf{s}}
$$

with usual multi-index notation, $\mathbf{s} !=s_{0} ! \ldots s_{r} !, \mathbf{y}^{\mathbf{s}}=y_{0}^{s_{0}} \cdots y_{r}^{s_{r}}$, and $\mathbf{T}^{\mathbf{s}}=$ $T_{0}^{s_{0}} \cup \ldots \cup T_{r}^{s_{r}}$. Consider its partial derivatives $\phi_{i j}=\frac{\partial^{2} \phi}{\partial y_{i} \partial y_{j}}=\sum_{\mathbf{s}} \frac{\mathbf{y}^{\mathbf{s}}}{\mathbf{s} !} \int_{X} \mathbf{T}^{\mathbf{s}} \cup$ $T_{i} \cup T_{j}$, and use the matrix $\left(g^{e f}\right)$ to raise indices, putting

$$
\phi_{j}^{i}=\sum_{e} g^{i e} \phi_{e j}, \quad \text { and } \quad \phi^{i j}=\sum_{e, f} g^{i e} \phi_{e f} g^{f j} .
$$

The entities $\phi_{j}^{i}(\mathbf{y})$ are the tensor elements of 'multiplication by the exponential', precisely

$$
\sum_{\mathbf{s}} \frac{\mathbf{y}^{\mathbf{s}}}{\mathbf{s} !} \mathbf{T}^{\mathbf{s}} \cup T_{p}=\sum_{e} T_{e} \phi_{p}^{e}(\mathbf{y}) .
$$

The deformed metric is the non-degenerate symmetric bilinear pairing $H^{*}(X, \mathrm{Q}) \rightarrow \mathrm{Q}[[\mathbf{y}]]$ given by the tensor elements

$$
\gamma_{i j}:=\phi_{i j}(-2 \mathbf{y}) \text {. }
$$

This concept was introduced in [10] as a tool for describing the way tangency conditions (or modified psi classes, cf. 1.4 below) restrict to the boundary of the moduli space of stable maps: the deformed metric arises as splitting factor for modified psi classes in the same way as the Poincaré metric does for primary Gromov-Witten invariants (incidence conditions).

The series $\phi$ satisfies the sum formula ([10], Lemma 2)

$$
\phi\left(\mathbf{y}^{\prime}+\mathbf{y}^{\prime \prime}\right)=\sum_{e, f} \phi_{e}\left(\mathbf{y}^{\prime}\right) g^{e f} \phi_{f}\left(\mathbf{y}^{\prime \prime}\right),
$$

where $\mathbf{y}^{\prime}$ and $\mathbf{y}^{\prime \prime}$ are formal variables. From this formula it follows that the inverse matrix of $\left(\gamma_{i j}\right)$ is given by

$$
\gamma^{i j}=\phi^{i j}(2 \mathbf{y})=\sum_{e, f} \phi_{e}^{i} g^{e f} \phi_{f}^{j} .
$$

We will also need certain derivatives of this,

$$
\gamma_{k}^{i j}:=\phi_{k}^{i j}(2 \mathbf{y})=\sum_{e, f} \phi_{e}^{i} g_{k}^{e f} \phi_{f}^{j} .
$$




\subsection{Moduli of stable maps}

Let $\bar{M}_{0, S}(X, \beta)$ denote the moduli stack of Kontsevich stable maps of genus zero whose direct image in $X$ is of class $\beta \in H_{2}^{+}(X, Z)$, and whose marking set is $S=\left\{p_{1}, \ldots, p_{n}\right\}$. For each mark $p_{i}$, let $v_{i}: \bar{M}_{0, S}(X, \beta) \rightarrow X$ denote the evaluation morphism. The reader is referred to Fulton-Pandharipande [6] for definitions and basic properties of stable maps, Gromov-Witten invariants and quantum cohomology. We use the word 'twig' for the irreducible components of a genus- 0 curve, reserving the term 'component' for the components of the moduli space.

\subsection{Modified psi classes and diagonal classes}

(cf. [GKP]). The psi class $\psi_{i}$ is the first Chern class of the line bundle on $\bar{M}_{0, S}(X, \beta)$ whose fibre at a moduli point $[\mu: C \rightarrow X]$ is the cotangent line of $C$ at $p_{i}$. On a moduli space $\bar{M}_{0, S}(X, \beta)$ with $\beta>0$, let $\xi_{i}$ denote the sum of all boundary divisor classes having mark $p_{i}$ on a contracting twig (= twig of degree 0 ). The modified psi class is defined as

$$
\bar{\psi}_{i}:=\psi_{i}-\xi_{i} \text {. }
$$

A crucial observation is that $\bar{\psi}_{i}$ is invariant under pull-back along forgetful morphisms.

The $i j$ 'th diagonal class $\delta_{i j}$ is by definition the sum of all boundary divisor classes having $p_{i}$ and $p_{j}$ together on a contracting twig. The diagonal classes appear as correction terms when restricting a modified psi class to a boundary divisor $D$ both of whose twigs are of positive degree. If $D$ is the image of the gluing morphism

$$
\rho_{D}: \bar{M}_{0, S^{\prime} \cup\{x\}}\left(X, \beta^{\prime}\right) \times_{X} \bar{M}_{0, S^{\prime \prime} \cup\{x\}}\left(X, \beta^{\prime \prime}\right) \longrightarrow \bar{M}_{0, S}(X, \beta)
$$

then

$$
\rho_{D}^{*} \overline{\boldsymbol{\psi}}_{i}=\overline{\boldsymbol{\psi}}_{i}+\boldsymbol{\delta}_{i x},
$$

where $x$ denotes the gluing mark.

\subsection{The tangency quantum potential}

(cf. [GKP]). The integrals

$\left\langle\bar{\tau}_{k_{1}}\left(\gamma_{1}\right) \cdots \bar{\tau}_{k_{n}}\left(\gamma_{n}\right)\right\rangle_{\beta}:=\int \bar{\psi}_{1}^{k_{1}} \cup v_{1}^{*}\left(\gamma_{1}\right) \cup \cdots \cup \overline{\boldsymbol{\psi}}_{n}^{k_{n}} \cup v_{n}^{*}\left(\gamma_{n}\right) \cap\left[\bar{M}_{0, n}(X, \beta)\right]$

$\left(\gamma_{i} \in H^{*}(X, \mathrm{Q})\right)$ are called enumerative descendants. For the first enumerative descendants (exponent at most 1 on modified psi classes) we employ the notation

$$
\left\langle\overline{\boldsymbol{\tau}}_{0}^{\mathbf{a}} \overline{\boldsymbol{\tau}}_{1}^{\mathbf{b}}\right\rangle_{\beta}:=\left\langle\prod_{k=0}^{r}\left(\bar{\tau}_{0}\left(T_{k}\right)\right)^{a_{k}}\left(\bar{\tau}_{1}\left(T_{k}\right)\right)^{b_{k}}\right\rangle_{\beta} .
$$


Their generating function is called the tangency quantum potential:

$$
\Gamma(\mathbf{x}, \mathbf{y})=\sum_{\beta>0} \sum_{\mathbf{a}, \mathbf{b}} \frac{\mathbf{x}^{\mathbf{a}}}{\mathbf{a} !} \frac{\mathbf{y}^{\mathbf{b}}}{\mathbf{b} !}\left\langle\overline{\boldsymbol{\tau}}_{0}^{\mathbf{a}} \overline{\boldsymbol{\tau}}_{1}^{\mathbf{b}}\right\rangle_{\beta} .
$$

The tangency quantum potential satisfies the topological recursion relations

$$
\Gamma_{y_{k} x_{i} x_{j}}=\Gamma_{x_{k}\left(x_{i} x_{j}\right)}-\Gamma_{\left(x_{k} x_{i}\right) x_{j}}-\Gamma_{\left(x_{k} x_{j}\right) x_{i}}+\sum_{e, f} \Gamma_{x_{k} x_{e}} \gamma^{e f} \Gamma_{x_{f} x_{i} x_{j}} .
$$

Here, and in the sequel, subscripts on potentials denote partial derivatives, e.g. $\Gamma_{x_{i}}:=\frac{\partial}{\partial x_{i}} \Gamma$, and we set also

$$
\Gamma_{\left(x_{i} x_{j}\right)}:=\sum_{k=0}^{r} g_{i j}^{k} \Gamma_{x_{k}} \quad \text { and } \quad \Gamma_{\left(y_{i} x_{j}\right)}:=\sum_{k=0}^{r} g_{i j}^{k} \Gamma_{y_{k}} .
$$

\section{Slightly enriched first enumerative descendants}

\subsection{Pi classes}

Let $\Pi_{i}$ denote the sum of all codimension- 2 boundary classes whose middle twig has degree 0 and carries the mark $p_{i}$, while the two other twigs have positive degree. Clearly $\Pi_{i}$ is compatible with pull-back along forgetful morphisms.

LEMma 2.2. Let $\Delta$ be an irreducible component of $\Pi_{i}$, then

$$
\bar{\psi}_{i} \cdot \Delta=0
$$

Proof. Let $x^{\prime}$ and $x^{\prime \prime}$ denote the two attachment points on the middle twig of $\Delta$. Now restrict $\overline{\boldsymbol{\psi}}_{i}=\boldsymbol{\psi}_{i}-\boldsymbol{\xi}_{i}$ to the moduli space $M_{i}$ corresponding to the middle twig: the psi class $\psi_{i}$ restricts to give the corresponding psi class of the mark of the middle twig. Restricting $\xi_{i}$ to $M_{i}$ corresponds to breaking off a twig containing $p_{i}$ but not $x^{\prime}$ nor $x^{\prime \prime}$. In other words, the restriction of $\boldsymbol{\xi}_{i}$ is $\left(p_{i} \mid x^{\prime}, x^{\prime \prime}\right)$ on $M_{i}$, which is the well-known boundary expression for $\psi_{i}$, so altogether the restriction of $\overline{\boldsymbol{\psi}}_{i}$ is zero.

Let $P^{k}$ denote the generating function for top products of classes of type $\bar{\tau}_{0}\left(T_{i}\right)$ and $\bar{\tau}_{1}\left(T_{j}\right)$ and a single factor (say at the first mark) of type $\Pi_{1} \cup v_{1}^{*}\left(T_{k}\right)$. Precisely

$$
P^{k}(\mathbf{x}, \mathbf{y}):=\sum_{\beta>0} \sum_{\mathbf{a}, \mathbf{b}} \frac{\mathbf{x}^{\mathbf{a}}}{\mathbf{a} !} \frac{\mathbf{y}^{\mathbf{b}}}{\mathbf{b} !}\left\langle\Pi_{1} \cup v_{1}^{*}\left(T_{k}\right) \overline{\boldsymbol{\tau}}_{0}^{\mathbf{a}} \overline{\boldsymbol{\tau}}_{1}^{\mathbf{b}}\right\rangle_{\beta} .
$$

(In view of Lemma 2.2, there is no reason for allowing also marks combining $\Pi$ with $\bar{\psi}$.) 
Proposition 2.3. The following differential equation relates $P^{k}$ to $\Gamma$ :

$$
P^{k}=\frac{1}{2} \sum_{e, f} \Gamma_{x_{e}} \gamma_{k}^{e f} \Gamma_{x_{f}} .
$$

Proof. Among the components of $\Pi_{1}$, those with at least two marks on the middle contracting twig have zero push-down under forgetting $p_{1}$, so we need only consider components of $\Pi_{1}$ where $p_{1}$ is alone on the middle twig. Each such component $\Delta$ is the image of a birational morphism from a triple fibred product $\bar{M}_{0, S \cup\left\{x^{\prime}\right\}}\left(X, \beta^{\prime}\right) \times_{X} \bar{M}_{0,3}(X, 0) \times_{X} \bar{M}_{0, S^{\prime \prime} \cup\left\{x^{\prime \prime}\right\}}\left(X, \beta^{\prime \prime}\right)$. Therefore there is the following sort of splitting lemma, similar to Lemma 1.5 of Kock [10]:

$$
\begin{aligned}
\left\langle\Delta \cdot v_{1}^{*}\left(T_{k}\right) \cdot \overline{\boldsymbol{\tau}}_{0}^{\mathbf{a}} \overline{\boldsymbol{\tau}}_{1}^{\mathbf{b}}\right\rangle_{\beta}=\sum_{\substack{p^{\prime}, q^{\prime} \\
p^{\prime \prime}, q^{\prime \prime}}} \sum_{\mathbf{s}^{\prime}, \mathbf{s}^{\prime \prime}}\left(\begin{array}{l}
\mathbf{b}^{\prime} \\
\mathbf{s}^{\prime}
\end{array}\right)\left(\begin{array}{l}
\mathbf{b}^{\prime \prime} \\
\mathbf{s}^{\prime \prime}
\end{array}\right)\left\langle\overline{\boldsymbol{\tau}}_{0}^{\mathbf{a}^{\prime}} \overline{\boldsymbol{\tau}}_{1}^{\mathbf{b}^{\prime}-\mathbf{s}^{\prime}} \bar{\tau}_{0}\left(\mathbf{T}^{\mathbf{s}^{\prime}} \cup T_{p^{\prime}}\right)\right\rangle_{\beta^{\prime}} \\
g^{p^{\prime} q^{\prime}} g_{q^{\prime} k p^{\prime \prime}} g^{p^{\prime \prime} q^{\prime \prime}}\left\langle\bar{\tau}_{0}\left(\mathbf{T}^{\mathbf{s}^{\prime \prime}} \cup T_{q^{\prime \prime}}\right) \overline{\boldsymbol{\tau}}_{0}^{\mathbf{a}^{\prime \prime}} \overline{\boldsymbol{\tau}}_{1}^{\mathbf{b}^{\prime \prime}-\mathbf{s}^{\prime \prime}}\right\rangle_{\beta^{\prime \prime}} .
\end{aligned}
$$

Translating this into a statement about the potentials yields the wanted differential equation. It is perhaps opportune to explain the appearance of the splitting factor $\gamma_{k}^{e f}$. At the gluing mark $x^{\prime}$ on the one-primed twig there is (after expressing things in terms of potentials) a factor $\sum_{\mathbf{s}^{\prime}} \frac{\mathbf{y}^{s^{\prime}}}{\mathbf{s}^{\prime}} \mathbf{T}^{\mathbf{s}^{\prime}} \cup T_{p^{\prime}}=\sum_{e} T_{e} \phi_{p^{\prime}}^{e}$, cf. (2). Arguing similarly on the two-primed twig we conclude that the splitting factor is

$$
\sum \phi_{p^{\prime}}^{e}\left(g^{p^{\prime} q^{\prime}} g_{q^{\prime} k p^{\prime \prime}} g^{p^{\prime \prime} q^{\prime \prime}}\right) \phi_{q^{\prime \prime}}^{f}=\sum \phi_{p^{\prime}}^{e} g_{k}^{p^{\prime} q^{\prime \prime}} \phi_{q^{\prime \prime}}^{f}=\gamma_{k}^{e f} .
$$

Note the presence of the factor $\frac{1}{2}$, due to the fact that all the components of $\Pi$ appear twice in the sum, depending on which of the two outer twigs we consider to be the one-primed and which is two-primed. In the very special case where $p_{1}$ is the only mark in play, there is no repetition in the sum since nothing distinguishes the two twigs, but this very symmetry means that the morphism from the fibred product is actually two-to-one, so in this case we divide by two for this reason.

Let $Q^{k}(\mathbf{x}, \mathbf{y})$ denote the generating function corresponding to first enumerative descendants allowing a single quadratic modified psi class, say at the first mark:

$$
Q^{k}(\mathbf{x}, \mathbf{y}):=\sum_{\beta>0} \sum_{\mathbf{a}, \mathbf{b}} \frac{\mathbf{x}^{\mathbf{a}}}{\mathbf{a} !} \frac{\mathbf{y}^{\mathbf{b}}}{\mathbf{b} !}\left\langle\overline{\boldsymbol{\tau}}_{0}^{\mathbf{a}} \overline{\boldsymbol{\tau}}_{1}^{\mathbf{b}} \bar{\tau}_{2}\left(T_{k}\right)\right\rangle_{\beta} .
$$


Proposition 2.4. The following differential equation relates $Q^{k}$ to the tangency quantum potential:

$$
Q_{x_{i} x_{j}}^{k}=\Gamma_{\left(x_{i} x_{j}\right) y_{k}}-\Gamma_{\left(y_{k} x_{i}\right) x_{j}}-\Gamma_{\left(y_{k} x_{j}\right) x_{i}}+\sum_{e, f}\left(\Gamma_{y_{k} x_{e}}+\Gamma_{\left(x_{k} x_{e}\right)}\right) \gamma^{e f} \Gamma_{x_{f} x_{i} x_{j}} .
$$

Proof. The proof is similar to the proof of Equation (3) (see [GKP], 2.1.1 and [10], 3.4.) Let the mark $p_{1}$ correspond to the class $\bar{\tau}_{2}\left(T_{k}\right)$, and let $p_{2}$ and $p_{3}$ carry the extra classes $\bar{\tau}_{0}\left(T_{i}\right)$ and $\bar{\tau}_{0}\left(T_{j}\right)$ corresponding to the derivatives. Take one of the two modified psi classes $\bar{\psi}_{1}$ and write it as sum of boundary divisors, to each of which the remaining factors are restricted. The first three terms correspond to boundary divisors with trivial degree splitting; compared to Equation (3), they each have a derivative with respect to $y$ instead of $x$ because there is now one modified psi class left on $p_{1}$. As to the quadratic term, it splits up in two, because the factor $\bar{\psi}_{1} \cdot v_{1}^{*}\left(T_{k}\right)$ restricts to give $\bar{\psi}_{1}$. $v_{1}^{*}\left(T_{k}\right)+\delta_{1 x^{\prime}} \cdot v_{x^{\prime}}^{*}\left(T_{k}\right)$, sending the evaluation class $v_{1}^{*}\left(T_{k}\right)$ over to the gluing mark $x^{\prime}$. This explains the factor $\left(\Gamma_{y_{k} x_{e}}+\Gamma_{\left(x_{k} x_{e}\right)}\right)$ in the quadratic term.

Observe that $\sum \Gamma_{x_{e}} \gamma_{k}^{e f}=\sum \Gamma_{\left(x_{k} x_{m}\right)} \gamma^{m f}$, so the last quadratic term is very similar to the terms of $P_{x_{i} x_{j}}^{k}$.

For $k=0$, there is a much simpler equation:

COROllary 2.5 .

$$
Q^{0}=-\frac{1}{2} \sum_{e, f} \Gamma_{x_{e}} \gamma^{e f} \Gamma_{x_{f}}
$$

Proof. After using the dilaton equation $\Gamma_{y_{0}}=-2 \Gamma$ twice, the equation of the proposition reads

$$
Q_{x_{i} x_{i}}^{0}=-2 \Gamma_{\left(x_{i} x_{i}\right)}-2 \Gamma_{y_{i} x_{i}}-\sum_{e, f} \Gamma_{x_{e}} \gamma^{e f} \Gamma_{x_{f} x_{i} x_{i}}
$$

Now apply topological recursion to the second term and simplify, ending up with

$$
Q_{x_{i} x_{i}}^{0}=-\sum_{e, f} \Gamma_{x_{i} x_{e}} \gamma^{e f} \Gamma_{x_{f} x_{i}}-\sum_{e, f} \Gamma_{x_{e}} \gamma^{e f} \Gamma_{x_{f} x_{i} x_{i}}
$$

Integrating twice with respect to $x_{i}$ yields the result.

REMARK 2.6. It is immediate from the formulae that $P^{0}+Q^{0}=0$. In fact, more generally, the classes $-\Pi_{1}$ and $\bar{\psi}_{1}^{2}$ on one-pointed space $\bar{M}_{0,1}(X, \beta)$ have the same push-down in $\bar{M}_{0,0}(X, \beta)$. Indeed, generally $\Pi_{1}$ pushes down to give the whole boundary. On the other hand, the push-down of $\bar{\psi}_{1}^{2}=\boldsymbol{\psi}_{1}^{2}$ 
is the kappa class by definition (see Arbarello-Cornalba [2]), and according to Pandharipande [13], Lemma 2.1.2, the kappa class is minus the boundary. (That proof treats $\mathrm{P}^{r}$ but it carries over to the present case.)

\section{Cuspidal curves in $P^{2}$}

In this section we consider $X=\mathrm{P}^{2}$, with its usual cohomology basis $\left(T_{0}=\right.$ fundamental class, $T_{1}=$ line, $T_{2}=$ point $)$. Set $\boldsymbol{\eta}_{i}:=c_{1}\left(v_{i}^{*}\left(T_{1}\right)\right)$.

\subsection{The characteristic number potential}

(cf. [GKP] §4). Let $N_{d}(a, b, c)$ denote the number of irreducible plane rational curves of degree $d$ which pass through $a$ general points, are tangent to $b$ general lines, and are tangent to $c$ general lines at a specified point, and define the number to be zero unless $a+b+2 c=3 d-1$.

Let $\Omega, \Theta$, and $\Xi$ denote the classes corresponding to these three conditions, then (at mark $p_{1}$, say) we have

$$
\Omega=\eta_{1}^{2}, \quad \Theta=\eta_{1}\left(\eta_{1}+\bar{\psi}_{1}\right), \quad \text { and } \quad \Xi=\eta_{1}^{2} \bar{\psi}_{1} .
$$

The characteristic number potential

$$
G(s, u, v, w)=\sum_{d>0} \exp (d s) \sum_{a, b, c} \frac{u^{a}}{a !} \frac{v^{b}}{b !} \frac{w^{c}}{c !} N_{d}(a, b, c)
$$

is related to the tangency quantum potential $\Gamma$ by

$$
G(s, u, v, w)=\Gamma\left(x_{1}, x_{2}, y_{1}, y_{2}\right),
$$

subject to the change of variables:

$$
x_{1}=s, \quad x_{2}=u+v, \quad y_{1}=v, \quad y_{2}=w,
$$

(and for simplicity we set $x_{0}=y_{0}=0$ throughout). Equation (5) is the expression of the fact that the change-of-variables (6) is dual to (4).

For the deformed metric we have

$$
\left(\gamma^{e f}\right)=\left(\begin{array}{ccc}
0 & 0 & 1 \\
0 & 1 & 2 y_{1} \\
1 & 2 y_{1} & 2 y_{1}^{2}+2 y_{2}
\end{array}\right)=\left(\begin{array}{ccc}
0 & 0 & 1 \\
0 & 1 & 2 v \\
1 & 2 v & 2 v^{2}+2 w
\end{array}\right),
$$

so in terms of the two differential operators

$$
\begin{aligned}
& \mathscr{L}:=\frac{\partial}{\partial s}+2 v \frac{\partial}{\partial u}, \\
& \mathscr{P}:=2 v \frac{\partial}{\partial s}+\left(2 v^{2}+2 w\right) \frac{\partial}{\partial u},
\end{aligned}
$$


the topological recursion relations satisfied by the characteristic number potential can be written

$$
\begin{aligned}
G_{v s} & =G_{u s}-G_{u}+\frac{1}{2}\left(G_{s s} \cdot \mathscr{L} G_{s}+G_{u s} \cdot \mathscr{P} G_{s}\right), \\
G_{w s s} & =G_{u u}+\left(G_{u s} \cdot \mathscr{L} G_{s s}+G_{u u} \cdot \mathscr{P} G_{s s}\right) .
\end{aligned}
$$

\subsection{The slightly enriched potentials}

Combining Propositions 2.3 and 2.4 with the above coordinate changes, we can express the slightly enriched potentials in terms of the characteristic number potential. We have

$$
\left(\gamma_{1}^{e f}\right)=\left(\begin{array}{ccc}
0 & 0 & 0 \\
0 & 0 & 1 \\
0 & 1 & 2 y_{1}
\end{array}\right) \quad \text { and } \quad\left(\gamma_{2}^{e f}\right)=\left(\begin{array}{lll}
0 & 0 & 0 \\
0 & 0 & 0 \\
0 & 0 & 1
\end{array}\right) \text {, }
$$

so from Proposition 2.3 we get

$$
\begin{aligned}
& P_{x_{1} x_{1}}^{1}=G_{u s} G_{s s}+G_{u s s} G_{s}+G_{u} \cdot \mathscr{L} G_{s s}+G_{u s} \cdot \mathscr{L} G_{s}, \\
& P_{x_{1} x_{1}}^{2}=G_{u s} G_{u s}+G_{u s s} G_{u} .
\end{aligned}
$$

Here we have taken double derivative with respect to $x_{1}$, anticipating the applications.

Similarly, for the $Q$-potential, Proposition 2.4 gives these three equations:

$$
\begin{aligned}
Q^{0}=-\frac{1}{2}\left(G_{s} \cdot \mathscr{L} G+G_{u} \cdot \mathscr{P} G\right), & \\
Q_{x_{1} x_{1}}^{1}=G_{v u}- & 2 G_{w s}-G_{w s s}+G_{s} G_{u s s}+G_{u} \cdot \mathscr{L} G_{s s} \\
& \quad+\left(G_{v s} \cdot \mathscr{L} G_{s s}+G_{v u} \cdot \mathscr{P} G_{s s}\right), \\
Q_{x_{1} x_{1}}^{2}= & G_{w u}+G_{u} G_{u s s}+\left(G_{w s} \cdot \mathscr{L} G_{s s}+G_{w u} \cdot \mathscr{P} G_{s s}\right) .
\end{aligned}
$$

(In deriving (12), the chain rule enters non-trivially, producing five extra terms which are exactly minus the right hand side of Equation (8), which is then used backwards.)

\subsection{The locus of marked cusp}

In the space $M_{0,1}\left(\mathrm{P}^{2}, d\right)$ of irreducible maps with a single mark $p_{1}$, Consider the locus of maps such that $p_{1}$ is a critical point, i.e. the differential vanishes at $p_{1}$. The locus of non-immersions is of codimension 1 , so requiring further that the mark is critical gives codimension 2 . Let $\mathrm{K}_{1}$ denote the closure of this 
locus in $\bar{M}_{0,1}\left(\mathrm{P}^{2}, d\right)$, the locus of maps having a cusp at $p_{1}$. In spaces with more marks, $\mathrm{K}_{1}$ is defined as the pull-back of the one in $\bar{M}_{0,1}\left(\mathrm{P}^{2}, d\right)$ via the forgetful morphism.

Proposition 3.4. The class of this marked cusp locus is

$$
\mathrm{K}_{1}=3 \boldsymbol{\eta}_{1}^{2}+3 \boldsymbol{\eta}_{1} \overline{\boldsymbol{\psi}}_{1}+\overline{\boldsymbol{\psi}}_{1}^{2}-\Pi_{1} .
$$

Proof. We start out with a family of stable un-pointed maps

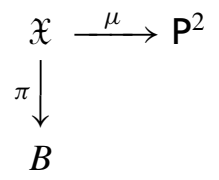

where $B$ and $\mathfrak{X}$ are smooth, and the locus $N \subset \mathfrak{X}$ of singular points of the fibres is of codimension 2. Let $I \subset \mathscr{O}_{\mathfrak{X}}$ be the ideal sheaf of $N$. The exact sequence

$$
0 \rightarrow \pi^{*} \Omega_{B} \rightarrow \Omega_{\mathfrak{X}} \rightarrow I \otimes \omega_{\pi} \rightarrow 0
$$

yields the relation of total Chern classes $c\left(T_{\mathfrak{x}}\right)=\pi^{*} c\left(T_{B}\right)\left(1-K_{\pi}+[N]\right)$, and thus

$$
\frac{\pi^{*} c\left(T_{B}\right)}{c\left(T_{\mathfrak{X}}\right)}=1+K_{\pi}+K_{\pi}^{2}-[N] .
$$

Here, $K_{\pi}:=c_{1}\left(\omega_{\pi}\right)$, and we also set $H:=\mu^{*} c_{1}(\mathscr{O}(1))$.

Denote temporarily by $D$ the class of the locus of points in $\mathfrak{X}$ where the differential $T_{\mathfrak{X}} \rightarrow(\pi \times \mu)^{*} T_{B \times \mathrm{P}^{2}}$ fails to have rank 2. By Porteous' formula, $D$ is the degree-2 part of the total Chern class

$$
\mu^{*} c\left(T_{\mathrm{P}^{2}}\right) \cdot \frac{\pi^{*} c\left(T_{B}\right)}{c\left(T_{\mathfrak{X}}\right)}=\left(1+3 H+3 H^{2}\right)\left(1+K_{\pi}+K_{\pi}^{2}-[N]\right),
$$

by (14). In other words,

$$
D=3 H^{2}+3 H K_{\pi}+K_{\pi}^{2}-[N]
$$

All this is basically $\S 4 . d$ of Diaz-Harris [4].

Now equip the family with a section $\sigma_{1}: B \rightarrow \mathfrak{X}$ that transversely intersects $N$. The marked-cusp class of the family is just $\mathrm{K}_{1}=\sigma_{1}^{*} D$. Now $\sigma_{1}^{*} H=\eta_{1}$ and $\sigma_{1}^{*} K_{\pi}=\psi_{1}=\bar{\psi}_{1}$, so we get

$$
\mathrm{K}_{1}=3 \boldsymbol{\eta}_{1}^{2}+3 \boldsymbol{\eta}_{1} \overline{\boldsymbol{\psi}}_{1}+\overline{\boldsymbol{\psi}}_{1}^{2}-\sigma_{1}^{*}[N] .
$$


(In a family with more sections, we must pull back these constructions; therefore the modified psi class is the correct one to use.) This family of marked maps is not stable, but there is a well-defined stabilisation. It only remains to notice that the locus $\sigma_{1}^{*}[N] \subset B$ of the unstable family is the same as $\Pi_{1}$ of the stabilised family.

\subsection{An alternative construction}

also given in [9], describes $\mathrm{K}_{1}$ as the locus of maps $\mu: C \rightarrow \mathrm{P}^{2}$ such that a whole pencil of lines in $\mathrm{P}^{2}$ are tangent to $\mu(C)$ at $\mu\left(p_{1}\right)$. In other words, it is the degeneracy locus of the map of vector bundles $\sigma_{1}^{*} V^{3} \rightarrow \sigma_{1}^{*} L^{2}$, where $V^{3}$ is the $\mu$-pull-back of the complete linear system $H^{0}\left(\mathrm{P}^{2}, \mathscr{O}(1)\right)$, and $L^{2}$ is the sheaf of first principal parts of $\mu^{*} \mathscr{O}(1)$. But then it is necessary to correct for $\Pi_{1}$ afterwards.

Remark 3.6. For $d=1$, the locus is empty, so in this case Porteous' formula yields the relation $3 \eta_{1}^{2}+3 \eta_{1} \bar{\psi}_{1}+\bar{\psi}_{1}^{2}=0$. Under the natural identification of $\bar{M}_{0,1}\left(\mathrm{P}^{2}, 1\right)$ with the incidence variety $I \subset \mathrm{P}^{2} \times \check{\mathrm{P}}^{2}$ of points and lines in $\mathrm{P}^{2}$, this relation is equivalent to the well-known relation $h^{2}+\breve{h}^{2}=h \check{h}$.

For $d=2$, the multiple-covers occur already in codimension 1 . On the other hand, there are no birational maps in degree 2 with a critical point, so for $d=2$ the locus $\mathrm{K}_{1}$ consists of all the double covers such that the mark is one of the ramification points.

For $d \geq 3$, the locus of multiple-covers is of codimension at least 2 , so the extra condition of having the mark as one of the ramification points prevents these curves from contributing. So in this case the locus $\mathrm{K}_{1}$ consists generically of birational maps.

\subsection{Further cusp conditions}

Consider the codimension-3 condition of the marked cusp mapping to a given line. The class $\mathrm{Kl}_{1}$ of this condition is obtained simply by cutting with $\eta_{1}$ :

$$
\mathrm{Kl}_{1}=3 \eta_{1}^{2} \overline{\boldsymbol{\psi}}_{1}+\eta_{1} \overline{\boldsymbol{\psi}}_{1}^{2}-\eta_{1} \Pi_{1}
$$

Similarly, the locus of maps with marked cusp mapping to a specified point is

$$
\mathrm{Kp}_{1}=\eta_{1}^{2} \overline{\boldsymbol{\psi}}_{1}^{2}-\eta_{1}^{2} \Pi_{1}
$$

These two loci can also be constructed by the approach of 3.5, replacing the complete linear system by smaller systems, cf. [9]. 


\subsection{Tangency conditions in cuspidal environment}

Suppose we are inside the locus $K_{1}$ and want to impose the condition of being tangent to a given line $L$ at another mark, say $p_{2}$. Since for the general map in $\mathrm{K}_{1}$, the differential vanishes only simply a $p_{1}$, the arguments of [GKP] 3.1 and 3.3 show that the locus of maps which are not transversal to $L$ at $p_{2}$ is reduced of class $\eta_{2}\left(\eta_{2}+\bar{\psi}_{2}\right)$. However, contrary to the case of nodal curves, this locus has two irreducible components. In addition to the locus of honest tangencies, there is a component consisting of maps such that the $p_{1}$-cusp maps to $L$ and the two marks have come together, i.e., $\mathrm{K}_{1} \cdot \boldsymbol{\eta}_{1} \cdot \boldsymbol{\delta}_{12}$. We do not want to count these maps as tangencies, so in conclusion, the class of $p_{2}$-tangency in $p_{1}$-cuspidal environment is

$$
\Theta_{2}^{\prime}=\eta_{2}\left(\eta_{2}+\bar{\psi}_{2}\right)-\eta_{1} \delta_{12}
$$

Similarly, in $p_{1}$-cuspidal environment the class of $p_{2}$-tangency to a given line at a specified point is

$$
\Xi_{2}^{\prime}=\eta_{2}^{2} \bar{\psi}_{2}-\eta_{1}^{2} \delta_{12}
$$

We can now apply these conditions iteratively, and the top intersections will be the characteristic numbers for cuspidal plane curves.

Using the generating functions for the slightly enriched first enumerative descendants, and their relation to the tangency potential, it is straightforward to derive differential equations determining the cusp characteristic numbers from the nodal ones. Let $C_{d}(a, b, c)$ denote the number of cuspidal plane curves passing through $a$ points, tangent to $b$ lines, and tangent to $c$ lines at specified points. Let $C L_{d}(a, b, c)$ be defined similarly but requiring the cusp to fall on a specified line, and let $C P_{d}(a, b, c)$ denote the numbers where the cusp is required to fall at a specified point. Let $K(s, u, v, w), K L(s, u, v, w)$ and $K P(s, u, v, w)$ be the corresponding generating functions (the formal variables being defined as in 3.1).

Proposition 3.9. The cusp potentials $K P, K L$, and $K$ are determined by the characteristic number potential $G$ through the following equations.

$$
\begin{gathered}
K P_{s s}=G_{w u}-G_{u s} G_{u s}+\left(G_{w s} \cdot \mathscr{L} G_{s s}+G_{w u} \cdot \mathscr{P} G_{s s}\right) \\
K L_{s s}=+G_{v u}+2 G_{w s s}-v K P_{s s}-2 G_{w s}-G_{u s} G_{s s}-G_{u s} \cdot \mathscr{L} G_{s} \\
\quad+\left(G_{v s} \cdot \mathscr{L} G_{s s}+G_{v u} \cdot \mathscr{P} G_{s s}\right),
\end{gathered}
$$

$$
K=3 G_{v}-v K L-\left(\frac{1}{2} v^{2}+w\right) K P-\left(G_{s} \cdot \mathscr{L} G+G_{u} \cdot \mathscr{P} G\right) .
$$


Proof. The main point is to eliminate the diagonal classes. In each term of the expansion of the top product, the diagonal class $\boldsymbol{\delta}_{1 i}$ is alone at mark $p_{i}$, so we can push down forgetting $p_{i}$. The push-down formula is simply $\pi_{i *} \boldsymbol{\delta}_{1 i}=1$ (cf. [GKP], 1.3.2.).

Since $\boldsymbol{\eta}_{1}^{3}=0$, and since all diagonal classes come accompanied by a factor $\eta_{1}$, only few diagonal class terms survive the expansion of the top product. In the presence of a factor $\operatorname{Kp}_{1}=\eta_{1}^{2}\left(\bar{\psi}_{1}^{2}-\Pi_{1}\right)$, all the diagonal classes of the top product vanish. Thus,

$$
K P(s, u, v, w)=\left(Q^{2}-P^{2}\right)\left(x_{1}, x_{2}, y_{1}, y_{2}\right) .
$$

Now take double derivative with respect to $s=x_{1}$ and apply Equations (13) and (10). This establishes (16).

In the integral corresponding to (17), since there is a factor $\eta_{1}$ in $\mathrm{Kl}_{1}$, there is room for at most one diagonal class in each term of the expansion. So we get

$$
d^{2} C L_{d}(a, b, c)=d^{2} \mathrm{~K} 1 \Omega^{a} \Theta^{\prime b} \Xi^{c}=d^{2} \mathrm{~K} 1 \Omega^{a} \Theta^{b} \Xi^{c}-d^{2} b \mathrm{Kp} \Omega^{a} \Theta^{b-1} \Xi^{c} .
$$

Here $\mathrm{Kp}_{1}$ arises as $\eta_{1} \cdot \mathrm{Kl}_{1}$. The last term explains $-v K P_{s s}$ in the formula. In the first term we plug in $\mathrm{Kl}_{1}=3 \boldsymbol{\eta}_{1}^{2} \overline{\boldsymbol{\psi}}_{1}+\boldsymbol{\eta}_{1} \overline{\boldsymbol{\psi}}_{1}^{2}-\boldsymbol{\eta}_{1} \Pi_{1}=3 \Xi_{1}+\boldsymbol{\eta}_{1}\left(\overline{\boldsymbol{\psi}}_{1}^{2}-\Pi_{1}\right)$. Thus

$$
K L_{s s}=-v K P_{s s}+3 G_{w s s}+\left(Q_{x_{1} x_{1}}^{1}-P_{x_{1} x_{1}}^{1}\right) .
$$

The result now follows from Equations (9) and (12).

Finally in the expansion of the integral corresponding to (18), we get $b$ terms corresponding to one diagonal class from $\Theta^{\prime}$, further $\left(\begin{array}{l}b \\ 2\end{array}\right)$ terms with two diagonal classes from $\Theta^{\prime}$, and finally $c$ terms with one diagonal class from $\Xi^{\prime}$ :

$$
\begin{aligned}
& C_{d}(a, b, c)=\mathrm{K} \Omega^{a} \Theta^{\prime b} \Xi^{\prime c}=\mathrm{K} \Omega^{a} \Theta^{b} \Xi^{c}-b \mathrm{~K} 1 \Omega^{a} \Theta^{b-1} \Xi^{c}+\left(\begin{array}{c}
b \\
2
\end{array}\right) \mathrm{Kp} \Omega^{a} \Theta^{b-2} \Xi^{c}-c \mathrm{Kp} \Omega^{a} \Theta^{b} \Xi^{c-1} \\
&=\mathrm{K} \Omega^{a} \Theta^{b} \Xi^{c}-b C L_{d}(a, b-1, c) \\
& \quad-\left(\begin{array}{c}
b \\
2
\end{array}\right) C P_{d}(a, b-2, c)-c C P_{d}(a, b, c-1) .
\end{aligned}
$$

The last three terms explain $-v K L-\left(\frac{1}{2} v^{2}+w\right) K P$ in the formula. The first term is expanded to

$$
\mathrm{K} \Omega^{a} \Theta^{b} \Xi^{c}=3 N_{d}(a, b+1, c)+\left(\bar{\psi}_{1}^{2}-\Pi_{1}\right) \Omega^{a} \Theta^{b} \Xi^{c},
$$

and this last term corresponds to $Q^{0}-P^{0}$ which is then expanded using Lemma 2.6 and Equation (11). 
These differential equations are very similar to the recursions used in Ernström-Kennedy [5] (found with completely different methods), and are presumably equivalent (modulo Equations (7) and (8)), but I have not been able to identify all the terms of their recursion.

Remark 3.10. Setting $v=w=0$ (corresponding to considering only incidence conditions) and then differentiating with respect to $s$ yields

$$
\begin{aligned}
K_{s} & =3 G_{v s}-\frac{\partial}{\partial s} G_{s}^{2} \quad(\bmod (v, w)) \\
& =3\left(G_{u s}-G_{u}+\frac{1}{2} G_{s s}^{2}\right)-\frac{\partial}{\partial s} G_{s}^{2} \quad(\bmod (v, w)),
\end{aligned}
$$

which is equivalent to the recursion of Proposition 5 in Pandharipande [13].

\section{Cuspidal curves in $P^{1} \times P^{1}$}

\subsection{Set-up for $\mathrm{P}^{1} \times \mathrm{P}^{1}$}

Let $T_{0}$ be the fundamental class; let $T_{3}$ be the class of a point; and let $T_{1}$ and $T_{2}$ be the hyperplane classes pulled back from the two factors. A curve of class $\beta$ is said to have bi-degree $\left(d_{1}, d_{2}\right)$, where $d_{1}=\int_{\beta} T_{1}$ and $d_{2}=\int_{\beta} T_{2}$. A curve of bi-degree $(1,0)$ is called a horizontal rule, and a curve of bi-degree $(0,1)$ a vertical rule.

Let $N_{\left(d_{1}, d_{2}\right)}(a, b, c)$ denote the characteristic numbers of irreducible rational curves in $\mathrm{P}^{1} \times \mathrm{P}^{1}$ of bi-degree $\left(d_{1}, d_{2}\right)$ passing through $a$ general points, tangent to $b$ general curves of bi-degree $(1,1)$, and tangent to $c$ such curves at a specified point. The classes corresponding to these three conditions are, respectively: $\Omega=\bar{\tau}_{0}\left(T_{3}\right), \Theta=2 \bar{\tau}_{0}\left(T_{3}\right)+\bar{\tau}_{1}\left(T_{1}\right)+\bar{\tau}_{1}\left(T_{2}\right)$, and $\Xi=\bar{\tau}_{1}\left(T_{3}\right)$.

Let $G\left(u_{1}, u_{2}, u, v, w\right)$ be the corresponding generating function $\left(u_{1}\right.$ and $u_{2}$ being the formal variables corresponding to the partial degrees $d_{1}$ and $d_{2}$ ). Then we have $G\left(u_{1}, u_{2}, u, v, w\right)=\Gamma\left(x_{1}, x_{2}, x_{3}, y_{1}, y_{2}, y_{3}\right)$, with $x_{1}=u_{1}$, $x_{2}=u_{2}, x_{3}=u+2 v ; y_{1}=v, y_{2}=v, y_{3}=w$. For convenience, put also

$$
s:=u_{1}+u_{2}
$$

the formal variable corresponding to $T_{1}+T_{2}$. We have

$$
\left(\gamma^{e f}\right)=\left(\begin{array}{cccc}
0 & 0 & 0 & 1 \\
0 & 0 & 1 & 2 y_{1} \\
0 & 1 & 0 & 2 y_{2} \\
1 & 2 y_{1} & 2 y_{2} & 4 y_{1} y_{2}+2 y_{3}
\end{array}\right)=\left(\begin{array}{cccc}
0 & 0 & 0 & 1 \\
0 & 0 & 1 & 2 v \\
0 & 1 & 0 & 2 v \\
1 & 2 v & 2 v & 4 v^{2}+2 w
\end{array}\right) .
$$


Define three differential operators corresponding to the three last lines of this matrix,

$$
\begin{aligned}
& \mathscr{L}_{1}:=\frac{\partial}{\partial u_{2}}+2 v \frac{\partial}{\partial u}, \\
& \mathscr{L}_{2}:=\frac{\partial}{\partial u_{1}}+2 v \frac{\partial}{\partial u}, \\
& \mathscr{P}:=2 v \frac{\partial}{\partial u_{1}}+2 v \frac{\partial}{\partial u_{2}}+\left(4 v^{2}+2 w\right) \frac{\partial}{\partial u},
\end{aligned}
$$

and for convenience put also

$$
\mathscr{L}:=\mathscr{L}_{1}+\mathscr{L}_{2}=\frac{\partial}{\partial s}+4 v \frac{\partial}{\partial u} .
$$

Equations (25) and (26) of [GKP] read

(20) $G_{w s s}=2 G_{u u}+\left(G_{u u_{1}} \cdot \mathscr{L}_{1} G_{s s}+G_{u u_{2}} \cdot \mathscr{L}_{2} G_{s s}+G_{u u} \cdot \mathscr{P}_{s s}\right)$.

\subsection{Differential equations for the slightly enriched potentials}

We have

$$
\left(\gamma_{(12)}^{e f}\right)=\left(\begin{array}{cccc}
0 & 0 & 0 & 0 \\
0 & 0 & 0 & 1 \\
0 & 0 & 0 & 1 \\
0 & 1 & 1 & 2 y_{1}+2 y_{2}
\end{array}\right) \quad \text { and } \quad\left(\gamma_{3}^{e f}\right)=\left(\begin{array}{cccc}
0 & 0 & 0 & 0 \\
0 & 0 & 0 & 0 \\
0 & 0 & 0 & 0 \\
0 & 0 & 0 & 1
\end{array}\right) \text {. }
$$

Now applying the coordinate changes to Propositions 2.3 and 2.4 we get:

$$
\begin{aligned}
P_{x_{1} x_{1}}^{(12)} & =G_{u s} G_{s s}+G_{u s s} G_{s}+G_{u} \cdot \mathscr{L}_{s s}+G_{u s} \cdot \mathscr{L} G_{s}, \\
P_{x_{1} x_{1}}^{3} & =G_{u s} G_{u s}+G_{u s s} G_{u}, \\
Q^{0}= & -\frac{1}{2}\left(G_{u_{1}} \cdot \mathscr{L}_{1} G+G_{u_{2}} \cdot \mathscr{L}_{2} G+G_{u} \cdot \mathscr{P} G\right), \\
Q_{x_{12} x_{12}}^{(12)}= & 2 G_{v u}-4 G_{w s}-2 G_{w s s}+G_{s} G_{u s s}+G_{u} \cdot \mathscr{L}_{s s} \\
& \quad+\left(G_{v u_{1}} \cdot \mathscr{L}_{1} G_{s s}+G_{v u_{2}} \cdot \mathscr{L}_{2} G_{s s}+G_{v u} \cdot \mathscr{P} G_{s s}\right), \\
Q_{x_{12} x_{12}}^{3}= & 2 G_{w u}+G_{u} G_{u s s} \\
& \quad+\left(G_{w u_{1}} \cdot \mathscr{L}_{1} G_{s s}+G_{w u_{2}} \cdot \mathscr{L}_{2} G_{s s}+G_{w u} \cdot \mathscr{P} G_{s s}\right) .
\end{aligned}
$$

The derivation of these formulae follows the same arguments as in 3.2. 


\subsection{Differential equations}

Deriving equations for the cusp potentials for $\mathrm{P}^{1} \times \mathrm{P}^{1}$ similar to those of 3.9 is now straightforward. Since the tangent bundle has total Chern class $1+2\left(T_{1}+T_{2}\right)+4 T_{3}$, the locus of cusp at mark $p_{1}$ is

$$
4 v_{1}^{*}\left(T_{3}\right)+2\left(v_{1}^{*}\left(T_{1}\right)+v_{1}^{*}\left(T_{2}\right)\right) \bar{\psi}_{1}+\bar{\psi}_{1}^{2}-\Pi_{1} .
$$

Let $K P$ be the potential corresponding to cusp mapping to a specified point, (and further $a$ conditions of passing through a point, $b$ conditions of being tangent to a $(1,1)$-curve, and $c$ conditions of tangenciating such a curve at a specified point). Then

$$
K P_{s s}=2 G_{w u}-G_{u s} G_{u s}+\left(G_{w u_{1}} \cdot \mathscr{L}_{1} G_{s s}+G_{w u_{2}} \cdot \mathscr{L}_{2} G_{s s}+G_{w u} \cdot \mathscr{P} G_{s s}\right) .
$$

Let $K L$ be the generating function for such characteristic numbers, but with the cusp mapping to a specified $(1,1)$-curve. Then

$$
\begin{aligned}
K L_{s s}=2 G_{v u} & -4 G_{w s}-2 v K P_{s s}+2 G_{w s s}-G_{u s} G_{s s}-G_{u s} \cdot \mathscr{L}_{s} \\
& +\left(G_{v u_{1}} \cdot \mathscr{L}_{1} G_{s s}+G_{v u_{2}} \cdot \mathscr{L}_{2} G_{s s}+G_{v u} \cdot \mathscr{P} G_{s s}\right) .
\end{aligned}
$$

And finally, let $K$ be the generating function for the characteristic numbers of cuspidal curves in $\mathrm{P}^{1} \times \mathrm{P}^{1}$, with the cusp varying freely. Then

$$
K=2 G_{v}-v K L-\left(v^{2}+w\right) K P-\left(G_{u_{1}} \cdot \mathscr{L}_{1} G+G_{u_{2}} \cdot \mathscr{L}_{2} G+G_{u} \cdot \mathscr{P} G\right) .
$$

\subsection{Enumerative significance}

A priori these numbers count also reducible curves, one of whose twigs is a multiple cover of a rule. In fact, already the locus $\mathrm{K}_{1}$ is not irreducible: it has a component for each boundary divisor corresponding to degree splitting $(m, n)=(i, 0)+(m-i, n)$. For each of these divisors, the one-primed twig is always a multiple-cover of a horizontal rule, and forcing the mark to a ramification point produces the 'cusp' already in codimension 2 . The other ramification points can then satisfy tangency conditions, giving contribution in the characteristic number. (Similarly of course for maps comprising a cover of a vertical rule.)

However, when there are no conditions on the cusp, all solutions are in fact irreducible curves. This happens because one degree of freedom (that of varying the position of the ramification point marked $p_{1}$ which counts as the cusp), is useless for the sake of satisfying tangency (or incidence) conditions, since we have already excluded the case where the tangency condition is fulfilled at $p_{1}$. Now the dimension count is easy: The multiple-cover twig has $2 i-2$ 
ramification points, of which $2 i-3$ can be used for satisfying tangency conditions. The twig can satisfy a single incidence condition (which completely fixes the support of the curve). The honest twig (of bi-degree $(m-i, n)$ ) can as usual satisfy $2 m-2 i+2 n-1$ conditions. Thus we get a total of $2 m+2 n-3$ degrees of freedom, while the number of conditions imposed is $2 m+2 n-2$. So no reducible curves can contribute.

The situation is different in the cases where the cusp is required to fall on a $(1,1)$-curve or at a point. In these cases, the cusp can account for one condition in addition to just being a cusp. For example, in the case where the cusp is required to fall on a given point: The given point fixes the rule supporting the image of the $p_{1}$-twig, and this must be a ramification point. Then further $2 i-3$ ramification points can account for as many tangency conditions. On the other twig there is room for $2 m-2 i+2 n-1$ conditions. Total: $2 m+2 n-4$, which is exactly the number of conditions imposed. So the potential $K P$ encodes also reducible curves. A similar observation applies to $K L$.

Correcting for these reducible curves is a case for the techniques described in [GKP] §5. Since the unwanted contribution are multiple covers, the correction terms involve the (genus zero) Hurwitz numbers.

\subsection{Hurwitz numbers and multiple-covers of a rule}

(Cf. [GKP] §5.) Consider $X=\mathrm{P}^{1}$ (with $T_{0}=$ fundamental class, $T_{1}=$ class of a point). The invariants $N_{d}(b)=\left\langle\bar{\tau}_{1}\left(T_{1}\right)^{b}\right\rangle_{d}^{\mathrm{Pl}}$ are the simple genus zero Hurwitz numbers (the number of $d$-sheeted coverings $\mathrm{P}^{1} \rightarrow \mathrm{P}^{1}$ simply ramified over $b=2 d-2$ given points). The corresponding potential,

$$
H(t, v)=\sum_{d>0} \exp (d t) \sum_{b} \frac{v^{b}}{b !} N_{d}(b) .
$$

satisfies the topological recursion relation $H_{v t}=v H_{t t} \cdot H_{t t}$.

For $\mathrm{P}^{1} \times \mathrm{P}^{1}$, the generating function for the covers of a horizontal rule is

$$
I\left(u_{1}, u, v, w\right)=u H_{u_{1}}+\left(v^{2}+w\right) H_{v},
$$

where $H=H\left(u_{1}, v\right)$ is the Hurwitz potential of (26). Indeed, the supporting rule for an $i$-sheeted map is fixed by either one incidence condition, two tangency conditions, or one flag condition. Once the supporting rule is fixed, the Hurwitz potential encodes the number of possible coverings. For the incidence condition, there are $i$ choices for the mark; this explains the factor $u H_{u_{1}}$. For the case of $2 i-1$ tangency conditions, the rule must pass through one of the two intersection point of two of the given curves. This gives $2 \cdot\left(\begin{array}{c}2 i-1 \\ 2\end{array}\right)$ choices 
for the supporting rule, explaining the term $2 \cdot \frac{v^{2}}{2 !} H_{v}$. Finally, for one flag condition and $2 i-3$ tangency conditions, the flag fixes the supporting rule and translates into an extra $v$ condition on the covering of that rule.

For coverings of a vertical rule, we similarly find the generating function

$$
J\left(u_{2}, u, v, w\right)=u H_{u_{2}}+\left(v^{2}+w\right) H_{v},
$$

with $H=H\left(u_{2}, v\right)$.

Now the correction term corresponding to the fake reducible cusps counted in $K P$ is described by:

Proposition 4.6. The generating functions $K^{\mathrm{irr}}, K L^{\mathrm{irr}}$, and $K P^{\mathrm{irr}}$ for the characteristic numbers of irreducible cuspidal curves in $\mathrm{P}^{1} \times \mathrm{P}^{1}$ are related to $K, K L$, and $K P$ like this:

$$
\begin{aligned}
K^{\mathrm{irr}} & =K, \\
K L^{\mathrm{irr}} & =K L-\left(I_{v u_{1}} \cdot \mathscr{L}_{1} G+J_{v u_{2}} \cdot \mathscr{L}_{2} G+\left(I_{v u}+J_{v u}\right) \cdot \mathscr{P} G\right), \\
K P^{\mathrm{irr}} & =K P-\left(I_{w u_{1}} \cdot \mathscr{L}_{1} G+J_{w u_{2}} \cdot \mathscr{L}_{2} G\right) .
\end{aligned}
$$

Proof. It has already been shown that $K^{\mathrm{irr}}=K$. For the others, the correction term has an $I$-part coming from multiple-covers of the horizontal rule and a $J$-part corresponding to covers of a vertical rule. Let us explain the $I$-part. The arguments of [GKP] $§ 5$ show that

$$
I_{u_{1}} \cdot \mathscr{L}_{1} G+I_{u} \cdot \mathscr{P} G
$$

is the generating function for the numbers of the reducible maps comprising a multiple-cover of a horizontal rule. In the present situation $p_{1}$ is a ramification point on the twig covering the horizontal rule. Therefore, requiring further that $p_{1}$ maps to a given line is equivalent to requiring tangency to that line at $p_{1}$, so the $I$-potentials in (29) acquire a derivative with respect to $v$. This accounts for the $I$-part of the correction term to $K L$. Concerning $K P$ : requiring the ramification point $p_{1}$ to map to a given point is equivalent to imposing a flag condition on $p_{1}$, so in this case the $I$-potentials in (29) acquire a derivative with respect to $w$. It remains to note that $I_{w u}=0$ since a multiple-cover of a rule cannot pass through two given general points.

EXAmple 4.7. Setting $v=w=0$ in the equation for $K$ yields an easy expression for the numbers $C_{(m, n)}$ of (irreducible) cuspidal curves in $\mathrm{P}^{1} \times \mathrm{P}^{1}$ of bi-degree $(m, n)$ that pass through $2 m+2 n-2$ general points, in terms of 
the corresponding numbers $N_{(m, n)}$ of nodal curves:

$$
\begin{aligned}
C_{(m, n)}=\frac{4(d-1)}{d} & N_{(m, n)} \\
& +\sum\left(\begin{array}{c}
2 d-2 \\
2 d^{\prime}-1
\end{array}\right) \frac{\left(m^{\prime} n^{\prime \prime}+n^{\prime} m^{\prime \prime}\right)\left(d^{\prime} d^{\prime \prime}-d\right)}{d} N_{\left(m^{\prime}, n^{\prime}\right)} N_{\left(m^{\prime \prime}, n^{\prime \prime}\right)},
\end{aligned}
$$

where the sum is over degree splittings $m^{\prime}+m^{\prime \prime}=m$ and $n^{\prime}+n^{\prime \prime}=n$. For short we have set $d=m+n, d^{\prime}=m^{\prime}+n^{\prime}$, and $d^{\prime \prime}=m^{\prime \prime}+n^{\prime \prime}$.

\section{Characteristic numbers of curves with a prescribed triple contact}

Let $V \subset X$ be a general, very ample hypersurface, given as the zero scheme of a section $f$ of a line bundle $L$, and set $\boldsymbol{\eta}_{i}:=c_{1}\left(v_{i}^{*} L\right)$.

\subsection{Components mapping into $V$}

Denote by $\mathrm{I}_{1}=\mathrm{I}_{1}(V)$ (the closure of) the locus of maps such that $p_{1}$ is on a non-contracting twig that maps entirely into $V$. Note that we define $\mathrm{I}_{1}$ only on set-theoretic level and not as a cycle. In general, this locus has irreducible components in various codimensions. The locus of irreducible such maps $\mathrm{I}_{1} \subset$ $M_{0, n}(X, \beta)$ is the zero scheme of a regular section of the vector bundle $\pi_{*} \mu^{*} L$, so the codimension is $\operatorname{dim} H^{0}\left(\mathrm{P}^{1}, \mu^{*} L\right)$ (or it is empty).

However, commonly the component consisting of irreducible such maps is not the one of lowest codimension: For example, if $X=\mathrm{P}^{r}$ and $V$ is a hypersurface of degree $z$, then the locus $\mathrm{I}_{i} \subset M_{0, n}\left(\mathrm{P}^{r}, d\right)$ is of codimension $d z+1$, while the locus of reducible such maps having $p_{i}$ on a twig of degree 1 is of codimension $z+2$. So for $d \geq 2$ or $z \geq 2$, the locus $\mathrm{I}_{1}$ is always of codimension at least 3 . The only situation in which we need to worry about codimension 2, is $d=z=1$ : in this case codim $\mathrm{I}_{1}=2$, and of course these maps are irreducible.

\subsection{Enumerative irrelevance of $\mathrm{I}_{1}$}

Note first of all that $\mathrm{I}_{1}$ is compatible with inverse image under forgetful morphisms, since in the definition we have excluded the case of $p_{1}$ belonging to a twig contracting to a point in $H$.

Now in the locus $\mathrm{I}_{1}$, the mark $p_{1}$ has not been fixed: it can be any point of the twig mapping into $V$, and since this twig is not just a contracting twig, different choices of where to put the mark on the twig are non-isomorphic. The consequence of this observation is that $\mathrm{I}_{1}$ has zero push-down under forgetting $p_{1}$ (whatever the multiplicities attributed to the components of it). This in turn implies the vanishing of any top product $\mathrm{I}_{1} \cdot A_{\neq 1}$, where $A_{\neq 1}$ is a product of pull-back-compatible classes belonging to other marks than $p_{1}$. 
With these remarks we are in position to describe the locus of maps having a triple contact with $V$. For the argument to work we must impose the following condition on the moduli space and on $V$ :

$(\star)$ The general map tangent to $V$ is only simply tangent to $V$.

The easiest example violating this condition is $\bar{M}_{0,1}\left(\mathrm{P}^{r}, 1\right)$ when $V$ is a hyperplane: every line tangent to $V$ is actually contained in $V$.

Proposition 5.3. With ( $\star$ ) satisfied, let $\mathrm{F}_{1}$ denote the closure of the locus of irreducible immersions having a triple contact to $V$ at the mark $p_{1}$. Then its class is

$$
\mathrm{F}_{1}=\eta_{1}\left(\boldsymbol{\eta}_{1}+\overline{\boldsymbol{\psi}}_{1}\right)\left(\boldsymbol{\eta}_{1}+2 \overline{\boldsymbol{\psi}}_{1}\right)-\left[\mathrm{I}_{1}\right]_{3}-\boldsymbol{\eta}_{1} \Pi_{1},
$$

where $\left[I_{1}\right]_{3}$ denotes the codimension-3 part of $\mathrm{I}_{1}$.

PRoOF. We can assume there is only one mark $p_{1}$. Consider the zero scheme of the section $\sigma_{1}^{*} \partial_{\pi}^{2} \mu^{*} f$ of the bundle of second jets, $\sigma_{1}^{*} J_{\pi}^{2} \mu^{*} L$. It follows from the standard jet short exact sequence that the class of this locus is

$$
c_{3}\left(\sigma_{1}^{*} J_{\pi}^{2} \mu^{*} L\right)=\eta_{1}\left(\eta_{1}+\overline{\boldsymbol{\psi}}_{1}\right)\left(\boldsymbol{\eta}_{1}+2 \overline{\boldsymbol{\psi}}_{1}\right) .
$$

It is the codimension-3 locus of maps such that the pull-back of $f$ vanishes to order 3 at $p_{1}$. If it happens isolated at $p_{1}$, then we are in $\mathrm{F}_{1}$. Note that there is no contribution from $p_{1}$-marked cusps: the locus of such cusps mapping to $V$ is of codimension 3, but in general the differential vanishes only simply and thus the first jet vanishes but not the second.

If $\mu^{*} f$ vanishes identically on the twig carrying $p_{1}$ then either this twig is of degree zero, and then we are at $\eta_{1} \Pi_{1}$ (or in higher codimension); or the twig is of positive degree, and that means it maps entirely inside $V$ and then we are in the locus $I_{1}$. (By the condition $(\star)$, no component of $I_{1}$ can have codimension lower than 3 . There may be components of higher codimension but since a section of a rank-3 vector bundle cannot vanish isolated along such a component, we conclude that these loci are in fact already contained in one of the codimension- 3 loci, which must then be $F_{1}$. In other words, such maps are limits of honest triple contacts.)

To establish multiplicity-1 along $\Pi_{1}$ it is enough to construct a 1-parameter family running inside the locus of maps tangent to $V$ at $p_{1}$, and check that the section of the second jet bundle vanishes with multiplicity 1 along the $\Pi_{1}$ of this family. For simplicity we perform the argument only in the case $X=\mathrm{P}^{2}$.

Let $B \subset \mathrm{A}^{1}$ be a small open neighbourhood of 0 , and consider the rational 
map

$$
\begin{aligned}
\mu: B \times \mathrm{P}^{1} & \longrightarrow \mathrm{P}^{2} \\
(b,[s: t]) & \longmapsto\left[\begin{array}{c}
b^{d} f \\
b(t-s)^{2}\left(b^{d-1} g+s^{m-1} t^{n-1}\right) \\
s^{m} t^{n}+b^{m} t^{d}+b^{n} s^{d}
\end{array}\right] .
\end{aligned}
$$

where $m+n=d$. As to the other symbols: $f$ is a homogeneous polynomial in $s, t$ of degree $d$, and $g$ is homogeneous of degree $d-2$, with roots distinct from $(t-s)$.

Equip the map with the constant section

$$
\sigma_{1}(b):=(b,[1: 1]) .
$$

The map has two base points in the central fibre $C$ (equation $(b=0)$ ), namely $[0: 1]$ and $[1: 0]$. (The rest of $C$ is contracted to the point $[0: 0: 1]$ in $\mathrm{P}^{2}$.) The map has been constructed such that a single blow-up resolves the map. The result is a family over $B$ of 1-pointed stable maps of degree $d$, whose central fibre is an element in $\Pi_{1}$ (with degree splitting $m+n=d$ ). Since we are interested only in what happens at the section, we can forget about the blow-up.

Let $V \subset \mathrm{P}_{[x: y: z]}^{2}$ be the line with equation $y=0$, then clearly all members of the family are tangent to $V$ at the first mark. More precisely, $\mu^{*} V=$ $2 \sigma_{1}+C+R$, where $R$ is some residual curve not passing through the point $(0,[1: 1])$. The coefficient 1 in front of $C$ is the exponent of $b$ in the second coordinate function. The vanishing of the second jet is read off in the family as the intersection of $\sigma_{1}$ with $\mu^{*} V-2 \sigma_{1}=C+R$. This is just the transverse intersection at $(1,[1: 1])$. Thus, $\Pi_{1}$ has multiplicity 1 .

REMARK 5.4. Note that we are not excluding the possibility that all maps in $\mathrm{F}_{1}$ are in fact contained in $V$. This happens for example in $\bar{M}_{0,1}\left(\mathrm{P}^{3}, 1\right)$ when $V \subset \mathrm{P}^{3}$ is a quadric surface.

Since the general map in $\mathrm{F}_{1}$ is not a cusp, we can now impose further tangency conditions without necessity of extra corrections. Thus we are in position to compute the characteristic numbers of rational curves having a triple contact with $V$.

EXAMPLE 5.5 (The projective plane). Fix a general curve $V \subset \mathrm{P}^{2}$ of degree $z$. Let $N F_{d}^{z}(a, b, c)$ denote the number of rational curves of degree $d \geq 2$ that have triple contact with $V$, are tangent to $b$ lines, tangent to $c$ lines at specified points, and furthermore pass through $a$ specified points, $(a+b+2 c=2 d-3)$. Then

$$
N F_{d}^{z}(a, b, c)=\int_{\bar{M}} \mathrm{~F}_{1} \Omega^{a} \Theta^{b} \Xi^{c} .
$$


When expanding the class of $F_{1}$, we can ignore $I_{1}$ since it's push-down is zero, so we find

$$
=3 z^{2} \Xi \cdot \Omega^{a} \Theta^{b} \Xi^{c}+2 z \boldsymbol{\eta}_{1} \overline{\boldsymbol{\psi}}_{1}^{2} \Omega^{a} \Theta^{b} \Xi^{c}-z \boldsymbol{\eta}_{1} \Pi_{1} \Omega^{a} \Theta^{b} \Xi^{c} .
$$

If we let $F^{z}$ be the generating function for these numbers we see that

$$
F^{z}(s, u, v, w)=3 z^{2} G_{w}(s, u, v, w)+2 z Q^{1}(\mathbf{x}, \mathbf{y})-z P^{1}(\mathbf{x}, \mathbf{y})
$$

subject to the usual change of variables, cf. 3.1. Now take double derivative with respect to $s$ and use the expressions for $P^{1}$ and $Q^{1}$ (given in Equations (12) and (9)):

$$
\begin{aligned}
& F_{s s}^{z}=z\left((3 z-2) G_{w s s}+\right. 2 G_{v u}-4 G_{w s}+G_{s} G_{u s s}+G_{u} \cdot \mathscr{L} G_{s s}-G_{u s} G_{s s} \\
&\left.-G_{u s} \cdot \mathscr{L} G_{s}+2\left(G_{v s} \cdot \mathscr{L} G_{s s}+G_{v u} \cdot \mathscr{P} G_{s s}\right)\right) .
\end{aligned}
$$

Perhaps the most interesting case is when $z=1$ so we are talking about curves with a specified flex line.

REMARK 5.6. Exploring the finer geometry of $\mathrm{P}^{2}$, through the space of stable lifts to its second Semple bundle, Colley-Ernström-Kennedy [3] have recently given a much more comprehensive formula. Not only does their formula allow any number of triple contact conditions to given curves, but the given curves are also allowed to have double points and cusps! The only drawback of their formula is that the given curves are not allowed to be lines or to have linear components.

Example 5.7 (The quadric surface. $X=\mathrm{P}^{1} \times \mathrm{P}^{1}$ ). Continuing the notation from Section 4, let $N F_{(m, n)}(a, b, c)$ be the number of rational curves of bidegree $(m, n)$ which have triple contact with a given curve of bi-degree $(1,1)$, are tangent to $b$ other curves of bi-degree $(1,1)$, tangent to $c$ such curves at specified points, and furthermore passing through $a$ specified points, $(a+b+$ $2 c=2 m+2 n-3)$.

Let $F$ be the generating function for these characteristic numbers. Then the arguments of the preceding example show that

$$
F=6 G_{w}+2 Q^{(12)}-P^{(12)}
$$

subject to the usual variable changes $x_{1}=u_{1}, x_{2}=u_{2}, x_{3}=u+2 v, y_{1}=v$, $y_{2}=v$, and $y_{3}=w$. Taking double derivative with respect to $s=u_{1}+u_{2}=$ $x_{1}+x_{2}$, and using Equations (24) and (21) we get the formula

$$
\begin{array}{r}
F_{s s}=2 G_{w s s}+4 G_{v u}-8 G_{w s}+G_{s} G_{u s s}+G_{u} \cdot \mathscr{L}_{s s}-G_{u s} G_{s s}-G_{u s} \cdot \mathscr{L} G_{s} \\
+2\left(G_{v u_{1}} \cdot \mathscr{L}_{1} G_{s s}+G_{v u_{2}} \cdot \mathscr{L}_{2} G_{s s}+G_{v u} \cdot \mathscr{P} G_{s s}\right),
\end{array}
$$


which effectively determines the characteristic numbers of curves in $\mathrm{P}^{1} \times \mathrm{P}^{1}$ with one triple contact to a $(1,1)$-curve, from the usual characteristic numbers.

\section{REFERENCES}

1. Aluffi, Paolo, The enumerative geometry of plane cubics. II. Nodal and cuspidal cubics, Math. Ann. 289 (1991), 543-572.

2. Arbarello, Enrico, and Cornalba, Maurizio, Combinatorial and algebro-geometric cohomology classes on the moduli spaces of curves, J. Algebraic Geom. 5 (1996), 705-749.

3. Colley, Susan Jane, Ernström, Lars, and Kennedy, Gary, Contact formulas for rational plane curves via stable maps, J. Algebraic Geom. 10 (2001), 365-396.

4. Diaz, Steven and Harris, Joe, Geometry of the Severi variety, Trans. Amer. Math. Soc. 309 (1988), 1-34.

5. Ernström, Lars, and Kennedy, Gary, Recursive formulas for the characteristic numbers of rational plane curves, J. Algebraic Geom. 7 (1998), 141-181.

6. Fulton, William, and Pandharipande, Rahul, Notes on Stable Maps and Quantum Cohomology, In J. Kollár, R. Lazarsfeld and D. Morrison, editors, Algebraic Geometry, Santa Cruz 1995, vol. 62, II of Proc. Sympos. Pure. Math., pp. 45-96, 1997.

7. Graber, Tom, Kock, Joachim, and Pandharipande, Rahul, Descendant invariants and characteristic numbers, Amer. J. Math. 124 (2002), 611-647.

8. Kleiman, Steven, and Speiser, Robert, Enumerative geometry of cuspidal plane cubics, In Proceedings of the Vancouver Conference in Algebraic Geometry, 1984., vol. 6 of Canad. Math. Soc. Proc., pp. 227-268, 1986.

9. Kock, Joachim, Tangency quantum cohomology and enumerative geometry of rational curves, $\mathrm{PhD}$ thesis, Recife, Brazil, March 2000.

10. Kock, Joachim, Tangency quantum cohomology, to appear in Compositio Math.

11. Kontsevich, Maxim, and Manin, Yuri I., Gromov-Witten classes, quantum cohomology, and enumerative geometry, Comm. Math. Phys. 164 (1994), 525-562.

12. Miret, Josep, and Xambó Descamps, Sebastià, Geometry of complete cuspidal cubics, In Algebraic Curves and Projective Geometry. Proceedings, Trento 1988., Lecture Notes in Math. 1389 (1989), 195-234.

13. Pandharipande, Rahul, Intersections of Q-divisors on Kontsevich's moduli space $\bar{M}_{0, n}\left(\mathrm{P}^{r}, d\right)$ and enumerative geometry, Trans. Amer. Math. Soc. 351 (1999), 1481-1505.

14. Sacchiero, Gianni, Numeri caratteristici delle cubiche piane cuspidali, Preprint, 1985.

15. Schubert, Hermann, Kalkül der abzählenden Geometrie, Teubner, Leipzip, 1879, Reprint, Springer-Verlag, Berlin, 1979.

16. Vakil, Ravi, Enumerative geometry of plane curves of low genus, Preprint.

DEPARTAMENTO DE MATEMÁTICA

UNIVERSIDADE FEDERAL DE PERNAMBUCO

CIDADE UNIVERSITÁRIA 50670-901

RECIFE - PE - BRAZIL

E-mail: jojo@dmat.ufpe.br 